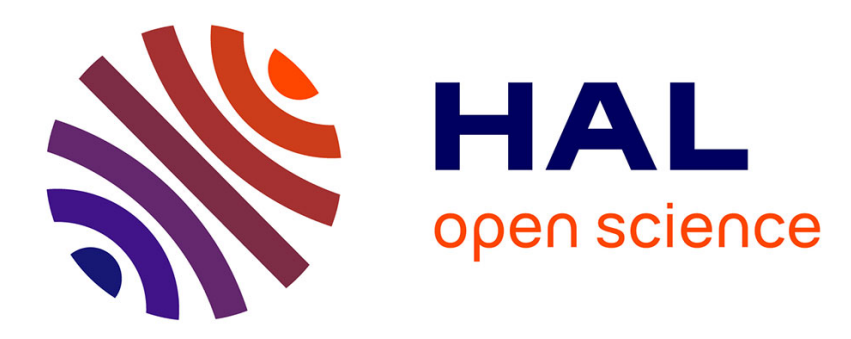

\title{
Les relations entre la ville et le fleuve à Paris de l'Antiquité gallo-romaine au Moyen Âge central
}

Hélène Noizet

\section{To cite this version:}

Hélène Noizet. Les relations entre la ville et le fleuve à Paris de l'Antiquité gallo-romaine au Moyen Âge central. Les Nouvelles de l'archéologie, 2011, 125, pp.32-40. halshs-00668548

\section{HAL Id: halshs-00668548 \\ https://shs.hal.science/halshs-00668548}

Submitted on 9 Feb 2012

HAL is a multi-disciplinary open access archive for the deposit and dissemination of scientific research documents, whether they are published or not. The documents may come from teaching and research institutions in France or abroad, or from public or private research centers.
L'archive ouverte pluridisciplinaire HAL, est destinée au dépôt et à la diffusion de documents scientifiques de niveau recherche, publiés ou non, émanant des établissements d'enseignement et de recherche français ou étrangers, des laboratoires publics ou privés. 


\section{Noizet, Hélène, "Les relations entre la ville et le fleuve à Paris de l'Antiquité gallo-romaine au Moyen Âge central", Les Nouvelles de l'archéologie, 2011, 125, p. 32-40.}

- Auteur : Hélène Noizet, université Paris-1 Panthéon-Sorbonne, LAMOP UMR 8589.

La récente mise en place d'un SIG sur l'espace parisien ancien (Noizet 2008-2009 ; Noizet Bove Costa à paraître) permet d'appréhender la dimension spatiale du processus d'urbanisation depuis l'Antiquité. Basée sur la reconstitution du plus ancien parcellaire de Paris (plans Vasserot 1810-1836), cette infrastructure géomatique ${ }^{1}$ permet de croiser la morphologie du tissu urbain (réseau viaire, parcelles, bâti) à des informations archéologiques et historiques de différentes époques, principalement médiévales et modernes : celles-ci donnent des éléments sur l'occupation humaine, qui a produit des espaces matériels (enceintes) ou idéels (censives, paroisses), mais également sur les caractéristiques physiques (topographie pré-urbaine, crues centennales). La structuration de l'espace urbain de Paris dans la longue durée peut donc être abordée en intégrant les différentes réalités du processus urbain, tant sociales qu'environnementales. Le parcellaire du début du XIX ${ }^{\mathrm{e}}$ siècle, avant les bouleversements haussmanniens, transmet des formes spécifiques, particulièrement curvilignes en rive droite. Celles-ci montrent que la relation à l'eau et les principaux flux de circulation se sont modifiés entre l'Antiquité et le Moyen Âge central, participant ainsi du basculement urbain de la rive gauche à la rive droite.

Nous observerons tout d'abord en quoi la relation à l'eau structure localement le tissu urbain en rive droite, puis nous montrerons que l'axe qui va, en diagonale, du port de la Grève aux Halles constitue, vers les $\mathrm{XI}^{\mathrm{e}}-\mathrm{XII}^{\mathrm{e}}$ s., une bifurcation durable du système viaire protohistorique et gallo-romain.

\section{1) Un rapport à l'eau structurant pour le tissu urbain de la rive droite}

Parmi les données géomorphologiques intégrées dans le SIG par Laurent Costa (fig. 1), figure la topographie pré-urbaine restituée par Vincent Krier lors de la fouille du Carrousel (Van Ossel 1998: 26-27) : à partir de l'Atlas géologique de la ville de Paris (Préfecture de la Seine 1967, 1/5000), celui-ci a retranché partout l'épaisseur des remblais modernes. Sa cartographie des courbes de niveau du relief ancien (en m NGF) met en exergue les dépressions inférieures à $30 \mathrm{~m}$ considérées comme zones inondables, en se fondant sur le relevé des cotes des crues décennales $(29 \mathrm{~m})$ et cinquantenaires (cote $31 \mathrm{~m})$. Mettant à jour le premier travail de reconstitution du sol dit « naturel » entrepris il y a plus d'un siècle (Villain 1895 ; Vallet 1910), ses apports sont pris en compte dans la Carte archéologique de la Gaule, notamment lorsqu'ils confirment ou affinent le récit géohistorique traditionnel de la rive droite (Busson 1998 : 50-53) : celui-ci fait des trois monceaux Saint-Germain l'Auxerrois, Saint-Merri, et Saint-Gervais des buttes naturelles qui auraient déterminé l'occupation humaine en rive droite à partir du haut Moyen Âge (Dion 1961 ; Rouleau 1988 : 40-41, carte I ; Busson 1998 : 53).

Cependant, D. Busson n'a pas relevé une discordance flagrante avec ce récit classique de la rive droite parisienne : non seulement le monceau Saint-Gervais, cité dans les textes dès 1137-1141 (Lasteyrie 1884: $\mathrm{n}^{\circ} 289$ et 292; Lombard-jourdan 1985: 81-82, 118 ;

\footnotetext{
${ }^{1}$ http://alpage.tge-adonis.fr/
} 
Bautier 1981 : 46), ne constitue pas un relief naturel sur la carte de V. Krier, mais il est même franchement une dépression qui forme une vaste zone inondable suivant approximativement les rues François Miron et Saint-Paul. Cette contradiction, qui ne peut résulter des inévitables déformations dues au géoréférencement du plan de V. Krier, reste à l'heure actuelle inexpliquée: dans le secteur de Saint-Gervais, une première vérification, faite par C. $\mathrm{Castanet}^{2}$, sur les données sources de V. Krier (atlas géologique de 1967) donne plutôt raison, dans le secteur de Saint-Gervais, à la CAG et aux restitutions anciennes qu'à celle de V. Krier.

Quoi qu'il en soit de cette difficulté locale, la géomorphologie restituée par V. Krier, à l'échelle plus grande du centre historique ancien, reste pertinente, notamment pour montrer la différence structurelle entre les deux rives, déjà relevée dans la bibliographie (Rouleau 1988 : 32, 40, carte I). La rive gauche se caractérise ainsi par une vaste zone humide, reculant l'espace non inondable à plus de $300 \mathrm{~m}$ au sud de l'actuelle berge, approximativement au niveau du boulevard Saint-Germain : de cette grande étendue marécageuse, un seul point haut émerge, Saint-Séverin, à partir duquel le réseau viaire se divise. A l'inverse, la rive droite se caractérise par une alternance d'anses et de reliefs bien marqués qui permettent de fixer plus près du fleuve non seulement des voies mais aussi l'occupation du sol proprement dite : mouille à l'est du Louvre (futur port de l'Ecole) jouxtant le monceau Saint-Germain, monceau Saint-Jacques-de-la-Boucherie jouxtant la mouille de la Grève.

L'influence de ces 2 mouilles s'est révélée durable pour la morphologie urbaine, mais de manière différente : la mouille de l'Ecole a longtemps marqué la limite de la ville médiévale alors que celle de la Grève en a constitué le centre. Une petite bande de $180 \mathrm{~m}$, centrée sur cette mouille de l'Ecole, et située entre l'église Saint-Germain 1'Auxerrois et la Cour carrée du Louvre, a d'abord attiré les débouchés dans le fleuve de l'enceinte du $\mathrm{X}^{\mathrm{e}}$ siècle et du rempart de Philippe Auguste (Noizet 2010, Noizet Bove Costa à paraître). Puis, suite au percement de la rue du Louvre à partir de 1853, dans la continuité de l'actuelle rue de Coligny, s'est formé, toujours dans ce secteur, une voie d'entrée importante en rive droite : cet axe de communication récent réactualise donc une discontinuité forte dans le tissu urbain.

Du côté de la Grève, l'influence morphologique de la mouille est très perceptible dans le réseau viaire et le parcellaire du début du XIX ${ }^{\mathrm{e}}$ siècle (fig. 2). On repère ainsi une forme en amande dans le tracé des anciennes rues de la Coutellerie et de la Tixeranderie, qui sont attestées au plus tard en 1300 et encore existantes telles quelles dans le premier tiers du XIX ${ }^{\mathrm{e}}$ s. Au nord, entre les îlots de la rue de la Tixeranderie et de la Verrerie, on retrouve un écho de cette forme remarquable dans les limites parcellaires et bâties à l'intérieur des îlots. Or, cette forme est concordante avec celle des courbes de niveaux du relief ancien restituées par T. Vacquer ${ }^{3}$.

Il nous semble que cette scansion morphologique résulte de la prégnance de la mouille de la grève, qui a durablement conditionné le tissu urbain dans ce secteur, avant que celui-ci ne soit profondément remodelé par le percement de la rue Rivoli en 1848-1854. L'accès à la Grève, et donc à la Seine, structure l'espace urbain de la rive droite.

\footnotetext{
${ }^{2}$ Nous remercions vivement Cyril Castanet de son analyse, qui reste à approfondir. Seule la constitution d'une base de données morphostratigraphiques, intégrant les différents niveaux sédimentaires des sondages, et modélisant des degrés d'incertitudes satisfaisants, permettra de résoudre la contradiction. Rappelons en effet que les fouilles du Carrousel avaient démenti un autre élément du discours géohistorique traditionnel, la zone basse du Louvre, qui se révèle en réalité être un bourrelet d'alluvions anciennes, insubmersible et sec (Van Ossel 1998: 26-39).

${ }^{3}$ Ici, contrairement au secteur de Saint-Gervais, les isohypses du paléorelief de T. Vacquer et V. Krier sont similaires, les divergences de forme ou de valeur restant mineures et relatives. Nous avons reporté les courbes de niveau de $\mathrm{T}$. Vacquer compte tenu des difficultés d'interprétation de la carte de V. Krier immédiatement à l'est de l'Hôtel de Ville.
} 
Dans la perspective du grand basculement urbain de la rive gauche à la rive droite, qui s'effectue au cours du haut Moyen Âge, on peut ainsi poser comme hypothèse qu'un changement culturel dans le rapport à l'eau prime sur les arguments militaire et économiques, déjà évoqués mais peu convaincants (Fleury 1974 : 38-39). Il ne s'agit pas de reprendre l'idée, effectivement critiquable, selon laquelle le changement de rive du site parisien serait dû au déplacement des grands épicentres politiques (en gros de la Méditerranée à la Mer du nord, ou de Rome à Aix-la-Chapelle), mais de souligner que le choix des populations parisiennes de mettre à profit les potentialités fluviales de la rive droite, à partir du haut Moyen Age, participe à expliquer le déplacement de la centralité urbaine du sud vers le nord.

Un premier avantage de la rive droite pour la navigation montante a été mis en valeur (Beaudoin 2000 : 46) : la concavité de la rive nord, en aval du port de Grève, conditionnant ainsi la présence du chenal profond et du chemin de halage sur la rive droite à l'arrivée dans Paris. Ensuite, les binômes reliefs/mouilles, décrits ci-dessus, offrent facilement la possibilité de s'installer au sec tout près de l'eau. Cette capacité à être à l'abri de l'eau devait se vérifier en régime hydrologique normal, mais également lors des crises fluviales : en cas de crues, il est probable que les débits liquides excédentaires aient été en partie absorbés par la dépression marécageuse héritée du grand paléoméandre, qui encerclait l'ensemble de la rive droite (de l'Arsenal au pont de l'Alma en passant aux pieds des collines de Belleville et Montmartre). Son rôle de chenal de défluviation est attesté à l'époque mérovingienne et a sans doute fonctionné au moins jusqu'au milieu du XII ${ }^{\mathrm{e}}$ siècle (Kleindienst 1963 ; Noizet Robert Mirlou à paraître). Au contraire, compte tenu des caractéristiques géographiques de la rive gauche (montagne Sainte-Geneviève dominant une vaste zone basse au contact du fleuve), la rive sud en bordure du fleuve devait être plus humide et ennoyée plus régulièrement lors des crues, d'autant plus qu'il n'existait pas de grand exutoire pour évacuer le trop plein des eaux.

La rive droite, qui était ainsi la plus adaptée pour vivre de manière pérenne au sec à proximité des zones d'accostage des bateaux, présente donc des atouts structurels plus favorables au commerce fluvial que la rive gauche. Or, force est de constater que, malgré l'importance du trafic fluvial gallo-romain et de la corporation des Nautes, ces éléments ne furent pas considérés comme décisifs à l'époque romaine, puisque les Romains ont fait le choix d'installer le centre de leur ville sur la Montagne Sainte-Geneviève, l'occupation urbaine n'ayant atteint la Seine que dans un second temps (Duval 1961). Si changement dans le rapport au fleuve il y a, la nature de ce changement serait donc plus culturelle que physique, dans la mesure où les caractéristiques géographiques, ici très grossièrement synthétisées, existaient autant à l'époque gallo-romaine qu'au Moyen Âge central : les sociétés qui se sont succédé sur le site parisien n'ont ainsi pas valorisé les mêmes potentialités physiques.

Par ailleurs, on observe qu'au cours du Moyen Âge, les inconvénients hydrologiques de la rive droite pour l'approvisionnement d'une population urbaine d'importance ont été compensés : contrairement à la rive gauche qui dispose de petits affluents de la Seine équipés d'infrastructures hydrotechniques telles que moulins et pêcheries (d'amont en aval : Bièvre, Petite Seine à Saint-Germain-des-Prés, rû du Bac), la rive droite ne dispose pas de ces écoulements pouvant facilement être artificialisés et favorables au développement d'un certain artisanat. Or, à partir des $\mathrm{IX}^{\mathrm{e}}-\mathrm{XI}^{\mathrm{e}}$ siècles, cette absence d'écoulements sur la rive droite a été compensée par des moulins utilisant directement l'eau du fleuve (moulins-bateaux) et accrochées aux ponts sur la Seine (moulins-pendants). Au XV siècle, la Seine ferait ainsi fonctionner une soixantaine de moulins, contre moins d'une dizaine pour la Bièvre (Berthier 2000). 
Un travail récent illustre a posteriori le rôle structurant du fleuve lui-même dans le tissu urbain de la rive droite : après avoir dépouillé toutes les données de la fouilles de la CVP et de l'INRAP dans les 4 premiers arrondissements, Léa Hermenault a spatialisé, dans le SIG d'ALPAGE, les vestiges archéologiques viaires de la protohistoire à la fin du Moyen Âge. Sa cartographie (fig. 3) montre que la presque totalité des vestiges archéologiques du réseau viaire médiéval concerne des structures qui offrent un accès au fleuve, parmi lesquelles 4 anciennes ruelles parallèles entre elles, une rampe d'accès au fleuve, une structure interprétée comme une "descente vers la Seine", et un niveau viaire perpendiculaire au fleuve (Hermenault 2010 : 101-102). Ces éléments du réseau viaire, qui datent principalement du bas Moyen Âge, sont donc littéralement branchés sur le fleuve. Or, le contraste est net avec les éléments du réseau viaire antique : parmi ceux retrouvés de ce secteur, aucun n'est lié à la Seine, excepté le cardo lui-même qui ne fait que traverser le fleuve. On ne peut arguer de l'insuffisance des données archéologiques (même si elles restent ténues) car les travaux pour les lignes 4 et $7 \mathrm{du}$ métro avaient été l'occasion de nombreuses fouilles en bord de Seine : or, elles n'avaient révélé aucun aménagement antique du même type que pour la période médiévale, alors même que la période antique a été privilégié dans certaines stratégies de fouilles parisiennes. Donc, même en faible nombre, les vestiges archéologiques de type viaire montre que les communications avec la Seine en rive droite étaient plus développées à la fin de la période médiévale qu'à l'époque gallo-romaine. Cette prépondérance des rues ayant une relation directe au fleuve à la fin du Moyen Âge confirme, a posteriori, que le rapport au fleuve de la population parisienne s'est transformé dans le sens d'un intérêt croissant pouvant expliquer le nouvel attrait de la rive droite au cours du haut Moyen Âge.

\section{2) La diagonale de la grève aux Halles, une bifurcation durable du système viaire protohistorique et gallo-romain}

Outre cette courbe des rues Tixeranderie-Coutellerie, le réseau viaire du centre ancien de la rive droite comporte d'autres spécificités morphologiques remarquables.

B. Rouleau avait déjà souligné la présence ancienne, sans doute proto-historique, du grand axe est-ouest, qui emprunte dans Paris les rues du Roi de Sicile, de la Verrerie, des Lombards et Saint-Honoré, et qui se poursuit, au niveau régional, vers Nanterre puis Rouen à l'ouest et vers Sens à l'est (fig. 4) : ce chemin rural, certainement antérieur à l'urbanisation de Paris, est en retrait des berges inondables, à environ 300-350 m au nord de l'actuelle Seine (Rouleau 1988: 47). Au nord de cet axe, le réseau viaire se développe de manière relativement orthogonale, avec un quadrillage articulé sur les deux voies d'origine romaine ouvrant vers le nord (rues Saint-Martin et Saint-Denis), tandis qu'au sud, la configuration en est très différente, essentiellement marqué par des limites viaires curvilignes. L'une d'elles, particulièrement longue, recoupe la forme en amande précédente et correspond à l'enfilades des rues médiévales suivantes : rue Jean de l'Espine, Jean Pain Mollet, des Ecrivains, de la Heaumerie, de la Cordouannerie et de la Limace. Ces rues, ainsi attestées dans le rôle de taille de 1300, existent encore au début du XIX ${ }^{\mathrm{e}}$ siècle, avec un même tracé et quasiment les mêmes noms (Bourlet à paraître). Cette enfilade fait se rejoindre la place de Grève et le sud de la rue Saint-Honoré, au sud des Halles.

En outre, le grand chemin protohistorique est-ouest est brutalement interrompu au niveau de Sainte-Opportune : la rue des Lombards s'arrête sur cette église et un décrochement important, vers le nord ou le sud, est nécessaire pour récupérer ce chemin au niveau de la rue Saint-Honoré (fig. 4). Si B. Rouleau notait que cette rupture était liée à l'enceinte des $\mathrm{X}^{\mathrm{e}}-\mathrm{XI}^{\mathrm{e}}$ s. de la rive droite, celui-ci ne relève pas qu'à l'autre extrémité de la rue interceptée par l'enceinte (rue du Bourg-Tibourg), il n'y a pas de rupture similaire à celle de Sainte- 
Opportune : à elle seule, la présence de cette enceinte ne suffit donc pas à rendre compte de cette rupture.

Enfin, une précédente analyse des orientations du parcellaire Vasserot a montré la prégnance de l'orientation romaine à $60-75^{\circ} \mathrm{E}$ (Robert à paraître) : cette trame parcellaire, dont le morphogène est l'ancien cardo romain (rues Saint-Jacques et Saint-Martin), structure aussi bien la rive gauche que la rive droite (fig. 5). Or, pour être dominante, cette orientation romaine, qui s'est principalement développée sur la rive droite à l'époque médiévale, n'est reste pas moins localement très discontinue, avec des absences particulièrement importantes (fig. 5) dans le secteur nord de la place de Grève, au sud de Saint-Jacques de la Boucherie et au sud-ouest de Sainte-Opportune. Ces ruptures suggèrent la concurrence d'une autre logique de circulation que celles héritées de l'occupation protohistorique et antique.

Ces trois spécificités morphologiques (l'enfilade curviligne des rues, le décrochement de Sainte-Opportune, les ruptures de la trame romaine) nous semblent s'expliquer par l'émergence d'un nouvel itinéraire structurant ce secteur, la liaison Grève-Champeaux. Cet itinéraire forme une diagonale, qui prend effectivement à rebours l'orientation romaine : il n'a pu émerger qu'à partir du moment où le port de la Grève et le marché des Halles sont devenus les principaux pôles d'attraction des flux en rive droite, soit pas avant le Moyen Âge central (Lombard-Jourdan 2009). Cet itinéraire a dû se frayer un chemin dans un espace urbain en cours de densification et dont les structures existantes renvoyaient à de toutes autres logiques de circulation, visant à longer et traverser la Seine.

Cet axe de circulation révèle aussi un autre point d'occupation important de la rive droite au cours du haut Moyen Âge : Saint-Jacques de la Boucherie. Cet établissement a manifestement attiré à lui cet itinéraire, contribuant ainsi à donner un aspect curviligne à cette diagonale, alors que Saint-Bon, déjà attestée du temps de saint Eloi (Duval et al. 1992 : 127), est délaissée au nord. Le premier état de Saint-Jacques de la Boucherie, qui remonte au $\mathrm{X}^{\mathrm{e}}$ siècle d'après T. Vacquer ${ }^{4}$, n'est pas antérieur au règne de Charles le Simple dans la mesure où une monnaie frappée à Melle au nom de ce souverain a été retrouvée, incorporée dans la maçonnerie même des substructions de l'édifice primitif. Il s'agit donc d'une église plus récente que Saint-Bon, cette dernière existant déjà du temps de saint Eloi. Quoi qu'il en soit, des fouilles récentes, menées entre octobre 2008 et janvier 2009, confirment l'ancienneté de la fonction artisanale de ce quartier de potiers (Lagarde 2011). Un important site de production céramique du $\mathrm{IX}^{\mathrm{e}}$ ou du début du $\mathrm{X}^{\mathrm{e}}$ siècle a en effet été mis au jour : les fosses à déchets, découvertes sous les allées du square actuel, contenaient pas moins d'une tonne de céramique carolingienne, essentiellement des ratés de cuisson.

La liaison avec le secteur des Saints-Innocents et des Halles se faisait, au niveau de l'enceinte des $\mathrm{XI}^{\mathrm{e}}-\mathrm{XI}^{\mathrm{e}}$ siècles, par deux voies quittant la rue de la Limace pour déboucher rue Saint-Honoré : la rue des Déchargeurs et la rue des Bourdonnais. Les carrefours formés dans la rue Saint-Honoré avec la rue des Déchargeurs puis avec la rue de la Lingerie (face au côté occidental du cimetière), servaient de places de marché bien connues à la fin du Moyen Âge, la place aux Chats et la vieille place aux pourceaux (Lombard-jourdan 2009 : 180).

$\mathrm{Au}$ vu de sa morphologie, cet axe n'est donc pas une surimposition planifiée (type boulevard de Louis XIV ou percée haussmannienne), mais résulte d'une évolution de la pratique socio-économique caractérisée par une réorientation des principaux flux de circulation. Si nous ne pouvons mesurer plus précisément la réification progressive de cet itinéraire entre l'Antiquité tardive et le XII ${ }^{\mathrm{e}}$ siècle, il n'en constitue pas moins une bifurcation efficace et durable du système viaire : non seulement cette liaison a réussi à reconfigurer le réseau préexistant, mais en plus sa morphologie resta inchangée durant tout l'Ancien Régime.

\footnotetext{
${ }^{4}$ BHVP, papiers Vacquer, ms. 237, fol. 323v.
} 
Sa forte résilience est due à la pérennité du fonctionnement économique parisien fondé sur la primauté de la Grève comme lieu de débarquement des produits et sur le monopole des Halles comme lieu des échanges. On peut représenter la résilience de cette bifurcation du réseau viaire sous la forme d'un corridor, en relevant toutes les parcelles plus ou moins exclues de l'orientation dominante, dans une logique de connexité à partir de l'axe viaire (fig. 6).

On ne sait pas précisément dater le début du fonctionnement des deux pôles aux extrémités de cette diagonale, la Grève et les Halles : il est probable que la Grève ait servi de port dès l'époque gallo-romaine (Capitan 1901 ; Benoit 2000), tandis que la reconnaissance royale du marché des Halles de 1136-1137 ne fait que sanctionner un marché existant depuis une période inconnue (Lombard-Jourdan 2099 : 15-19). Mais plusieurs éléments convergent pour dater la réification finale de cet itinéraire, telle que nous pouvons la repérer dans l'espace urbain du $\mathrm{XIV}^{\mathrm{e}}$ au $\mathrm{XIX}^{\mathrm{e}}$ siècle, au plus tôt du $\mathrm{XI}^{\mathrm{e}}$ siècle, et plus certainement de la première moitié du XII ${ }^{\mathrm{e}}$ siècle.

Tout d'abord, le début de la rue Jean de l'Epine, qui est le point de départ de l'itinéraire place de Grève, se démarque nettement par l'avancée de la rue de la Vannerie vers la place (fig. 2) : or, ce décrochement s'explique par la présence de l'eau jusqu'à cette hauteur jusqu'au $\mathrm{XI}^{\mathrm{e}}-\mathrm{XII}{ }^{\mathrm{e}}$ siècle. La présence d'un mouillage, suggérée par la cartographie de $\mathrm{T}$. Vacquer, a été vérifié archéologiquement place de l'Hôtel de Ville (Capitan 1901). Un sondage, creusé à peu près au milieu de la place (fig. 2), a révélé une couche de limon noir tourbeux avec de nombreux débris de végétaux aquatiques, et ne contenant que du matériel gallo-romain (débris de céramiques et retailles de cuirs). La couche tourbeuse atteste la présence d'un milieu humide calme, donc en retrait du chenal principal du fleuve, qui est exploité à l'époque gallo-romaine pour la tannerie, indiquant ainsi que la berge à cette époque devait se trouver un peu plus au nord sans que l'on sache où précisément. La couche susjacente ne comporte, elle, que des débris de constructions avec mortiers de chaux et plâtre, et de la céramique des $\mathrm{XI}^{\mathrm{e}}-\mathrm{XIII}^{\mathrm{e}}$ s.: elle témoigne donc, à cet endroit précis, d'une transformation médiévale de l'occupation, caractérisée par les progrès de l'espace urbain au détriment de la zone humide. Selon L. Capitan, l'absence totale de déchets céramiques des $\mathrm{XI}^{\mathrm{e}}-\mathrm{XIII}{ }^{\mathrm{e}}$ dans la couche tourbeuse suggère que ce secteur n'a été exondé que tardivement, au plus tôt à partir des $\mathrm{VI}^{\mathrm{e}}-\mathrm{VII}{ }^{\mathrm{e}}$ siècle et au plus tard aux $\mathrm{XI}^{\mathrm{e}}-\mathrm{XII}^{\mathrm{e}} \mathrm{s}$. comme le montre la couche supérieure. Jusqu'à hauteur de la rue de la Vannerie, on a donc encore les pieds dans l'eau jusqu'au $\mathrm{XI}^{\mathrm{e}}-\mathrm{XII}^{\mathrm{e}}$ siècles, expliquant ainsi le décrochement de la rue Jean de l'Epine.

Ensuite, à l'autre extrémité de la diagonale, l'enceinte primitive de la rive droite, observée archéologiquement 15 rue du Temple puis 140 rue de Rivoli (Prié 1997 ; Peixoto Celly 2011), a impliqué une logique de fermeture au moins jusqu'au $\mathrm{XI}^{\mathrm{e}}$ siècle inclus. Rue de Rivoli, à un premier état de l'enceinte, datable uniquement par un terminus ante quem de 997 998 (Noizet 2010), a succédé un deuxième état, correspondant à une réfection du fossé au $\mathrm{XI}^{\mathrm{e}}$ s. Le fossé d'enceinte n'a été transformé en voie qu'au XII ${ }^{\mathrm{e}}$ siècle, comme l'atteste des niveaux de voiries sommaires mais tout de même travaillés : cet axe n'est pas un simple sentier tracé par le piétinement, mais une voie constituée de matériaux rapportés, et comportant de nombreuses empreintes de pas de chevaux ou d'ânes. La logique de fermeture impliquée par l'enceinte, qui conserve donc sa fonction défensive jusqu'au $\mathrm{XI}^{\mathrm{e}}$ siècle inclus, rend peu probable la mise en place de l'itinéraire en diagonale avant le début du XII siècle. Un autre élément intéressant livré par la fouille de la rue de Rivoli réside dans la faible durée d'utilisation de cette voie fabriquée dans le fossé : elle disparaît en effet dans le courant du $\mathrm{XII}^{\mathrm{e}}$ siècle, au profit d'une carrière de sables exploitée jusqu'au XIII ${ }^{\mathrm{e}}$ s., ce secteur étant finalement colonisé par de l'habitat à partir de la fin du XIII ${ }^{\mathrm{e}}$ s. (hôtel de Pontis). La disparition rapide de cette voie observée archéologiquement est parfaitement cohérente avec l'absence de voie, en 1300 comme en 1810-1836, entre l'extrémité occidentale de la rue de la 
Limace et la rue des Fossés Saint-Germain-l'Auxerrois. Dans ce secteur entre la rue de l'Arbre-Sec et la rue des Bourdonnais, la voirie mise en place sur le fossé d'enceinte n'a pas constitué une modification durable des flux, contrairement au tracé qui aboutit à la rue de la Limace. Ceci confirme ainsi la prééminence des Halles comme point d'aboutissement de l'itinéraire.

Enfin, une dernière série d'arguments textuels convergent avec une datation de l'itinéraire en diagonale dans la première moitié du XII ${ }^{\mathrm{e}} \mathrm{s}$. Plusieurs actes royaux prouvent une forte réorganisation des lieux d'approvisionnement entre 1136 et 1142, qui officialisent en quelque sorte la repolarisation du tissu urbain selon cette diagonale (Lombard-Jourdan 2009 : 18-19) : reconnaissance par le roi Louis VI du marché des Halles avec partage des profits avec l'évêque en 1136-1137 (Lasteyrie $1884: \mathrm{n}^{\circ} 264,271$ ) ; maintien de la place de grève en 1141-1142 comme espace vide conformément aux souhaits de la population qui veut continuer d'y débarquer les marchandises (Lasteyrie $1884: \mathrm{n}^{\circ} 289$ ). Dans les diplômes, une spécialisation apparaît donc nettement entre la Grève, ce « vieux marché » qui doit rester le port de débarquement des produits, et les Halles, le «nouveau marché » qui va progressivement acquérir un quasi-monopole sur la vente des denrées.

Tous les indicateurs convergent donc pour dater de la première moitié du XII ${ }^{\mathrm{e}}$ siècle la réorganisation du réseau viaire de la rive droite.

Pour finir, il faut souligner que cette temporalité tardive explique la rupture du grand chemin protohistorique de la rue Saint-Honoré au seul niveau de Sainte-Opportune (et non pas au Bourg-Tibourg): le responsable en est le décalage temporel entre la fermeture de l'enceinte au plus tard fin $\mathrm{X}^{\mathrm{e}} \mathrm{s}$. et le développement de cette diagonale à partir de la première moitié du XII ${ }^{\mathrm{e}} \mathrm{s}$. La fermeture imposée par l'enceinte a d'abord favorisé l'expansion de l'habitat jusqu'à s'adosser à l'enceinte elle-même, et notamment autour de l'église SainteOpportune. Ce n'est que lorsque l'enceinte perd sa fonction défensive que la connexion viaire entre la rue Saint-Honoré et la rue des Lombards pourrait être rétablie, pas avant le début du $\mathrm{XII}^{\mathrm{e}} \mathrm{s}$. Mais alors il est trop tard : à cette époque, il y a vraisemblablement un habitat un peu dense dans le secteur de Sainte-Opportune, trop compliqué à éclater (il faudrait négocier et obtenir l'accord de chaque habitant et chaque propriétaire, ce qui est concrètement difficilement faisable lorsque leur nombre est important). Surtout, les préoccupations ont changé : désormais, ce qui compte, c'est l'accès au marché plus que la liaison est-ouest, qui est donc localement réaménagée en fonction de cette nouvelle contrainte de la liaison à assurer entre la Grève et les Halles. Une telle contrainte n'ayant pas son équivalent à l'est, du côté du Bourg-Tibourg, l'ancien chemin rural est-ouest peut, là, reprendre ses droits lorsque l'enceinte est progressivement abandonnée.

En conclusion, la prise en compte de la morphologie urbaine (réseau viaire, trame parcellaire), ainsi que le croisement des données archéologiques, textuelles et planimétriques permet de mieux cerner l'évolution de l'espace urbain sur la rive droite. Cette évolution est décisive puisqu'elle intervient à une époque charnière de l'histoire urbaine, qui a vu entre l'Antiquité et le Moyen Âge central, sa centralité basculer de la rive gauche à la rive droite. De nouveaux rapports au fleuve semblent participer de ce basculement et conditionnent également la transformation durable du système viaire. Cette capacité à proposer un nouveau discours historique repose aussi sur des acquis conceptuels, tels ceux de l'archéogéographie : décalages spatio-temporels, hybridation des formes, bifurcation de systèmes spatiaux, intentionnalité des acteurs intégrée dans une analyse en termes de pôle, flux et tracé (Chouquer 2007 ; Robert 2003). 


\section{Bibliographie}

Liste des figures

1. Relief antérieur à l'urbanisation d'après V. Krier

2. Héritages viaires et parcellaires de la mouille de la Grève

3. Structures archéologiques des réseaux viaires antique et médiéval

4. Principaux tracés viaires en rive droite

5. Rupture de la trame parcellaire héritée de l'Antiquité sur la rive droite

6. Discordance parcellaire de l'axe Grève-Halles 


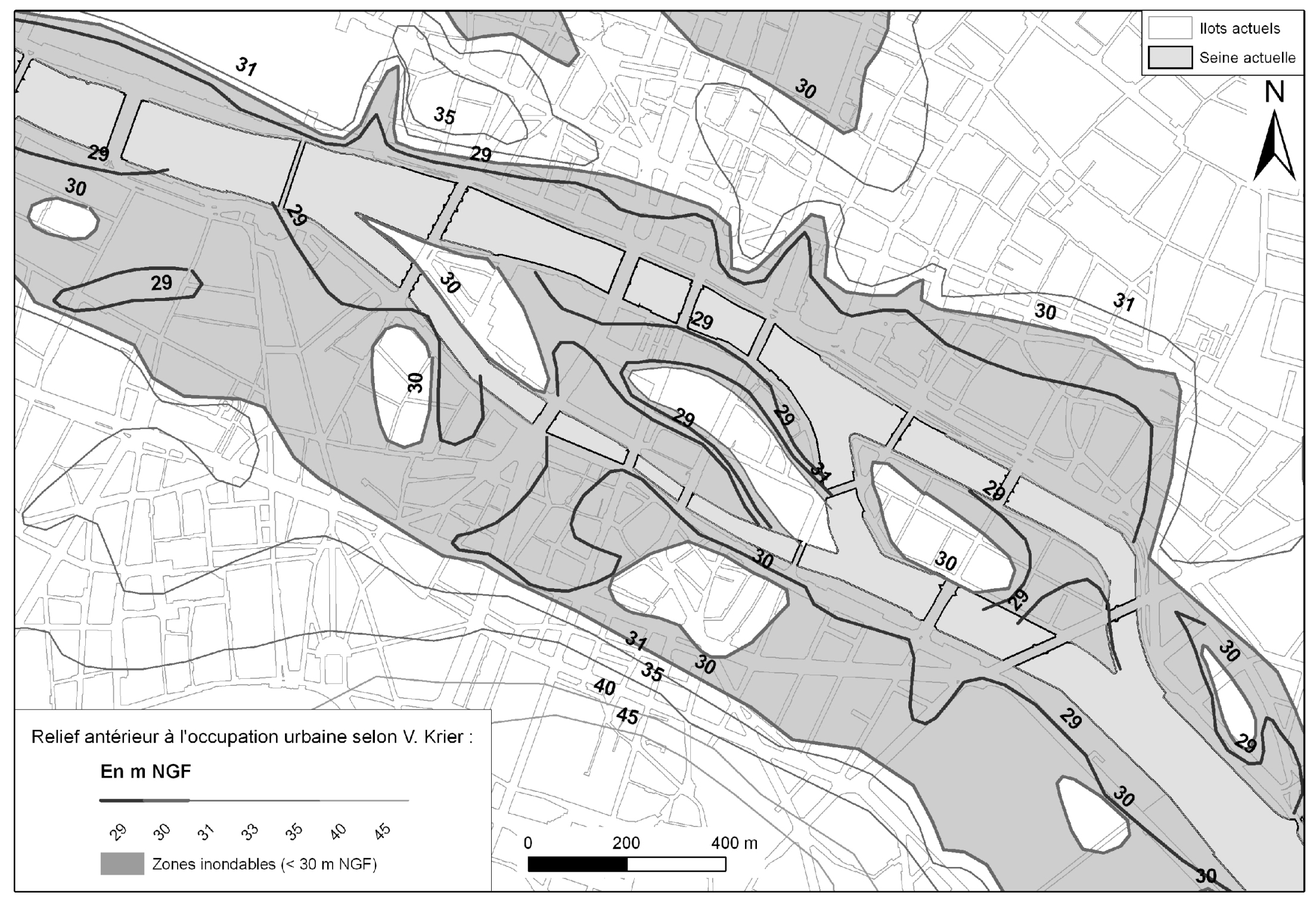

APUR @ ALPAGE : H. Noizet, L. Costa, V. Krier 1998 - HN, 2011

1. Relief antérieur à l'urbanisation d'après V. Krier 


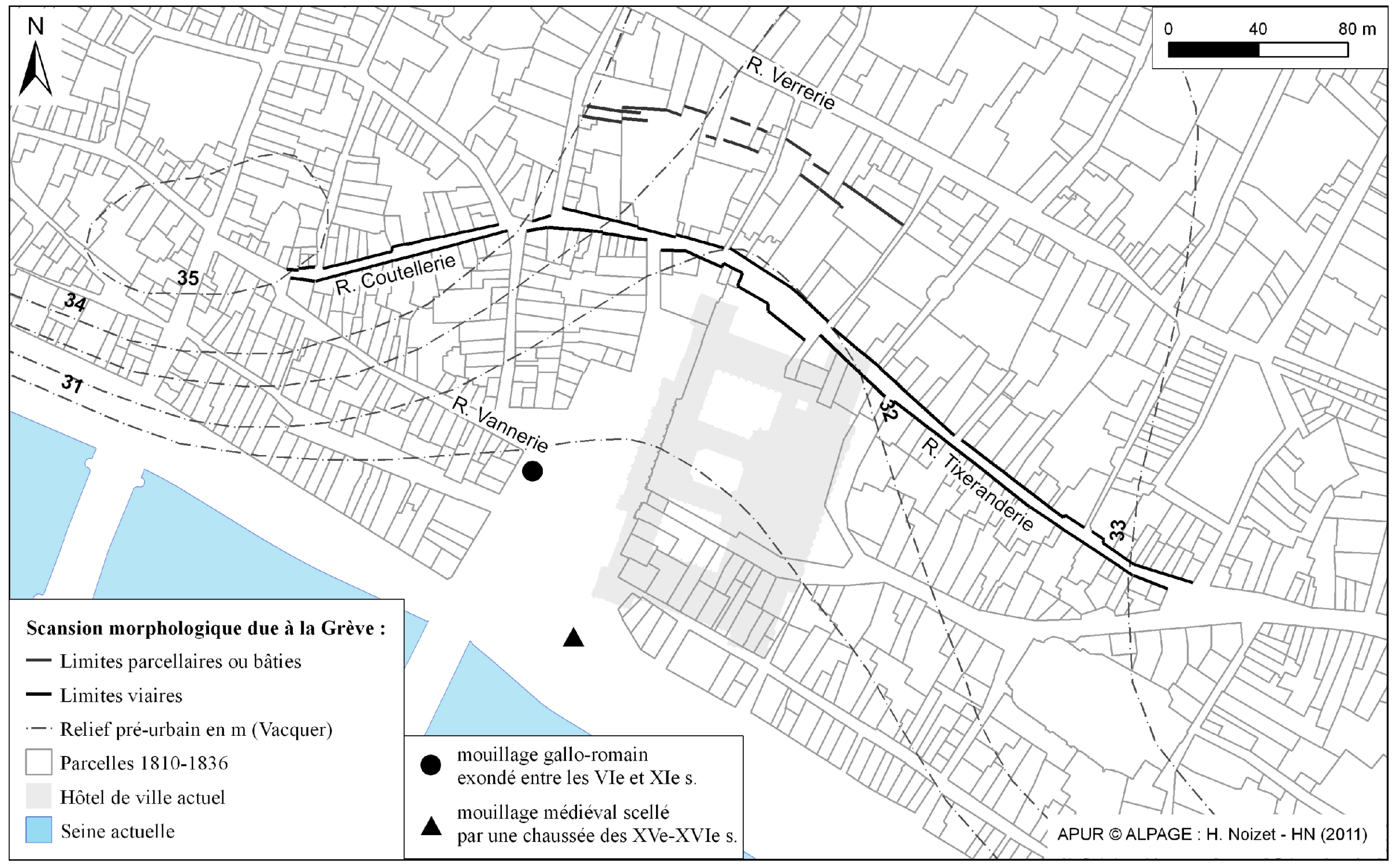

2. Héritages viaires et parcellaires de la mouille de la Grève 


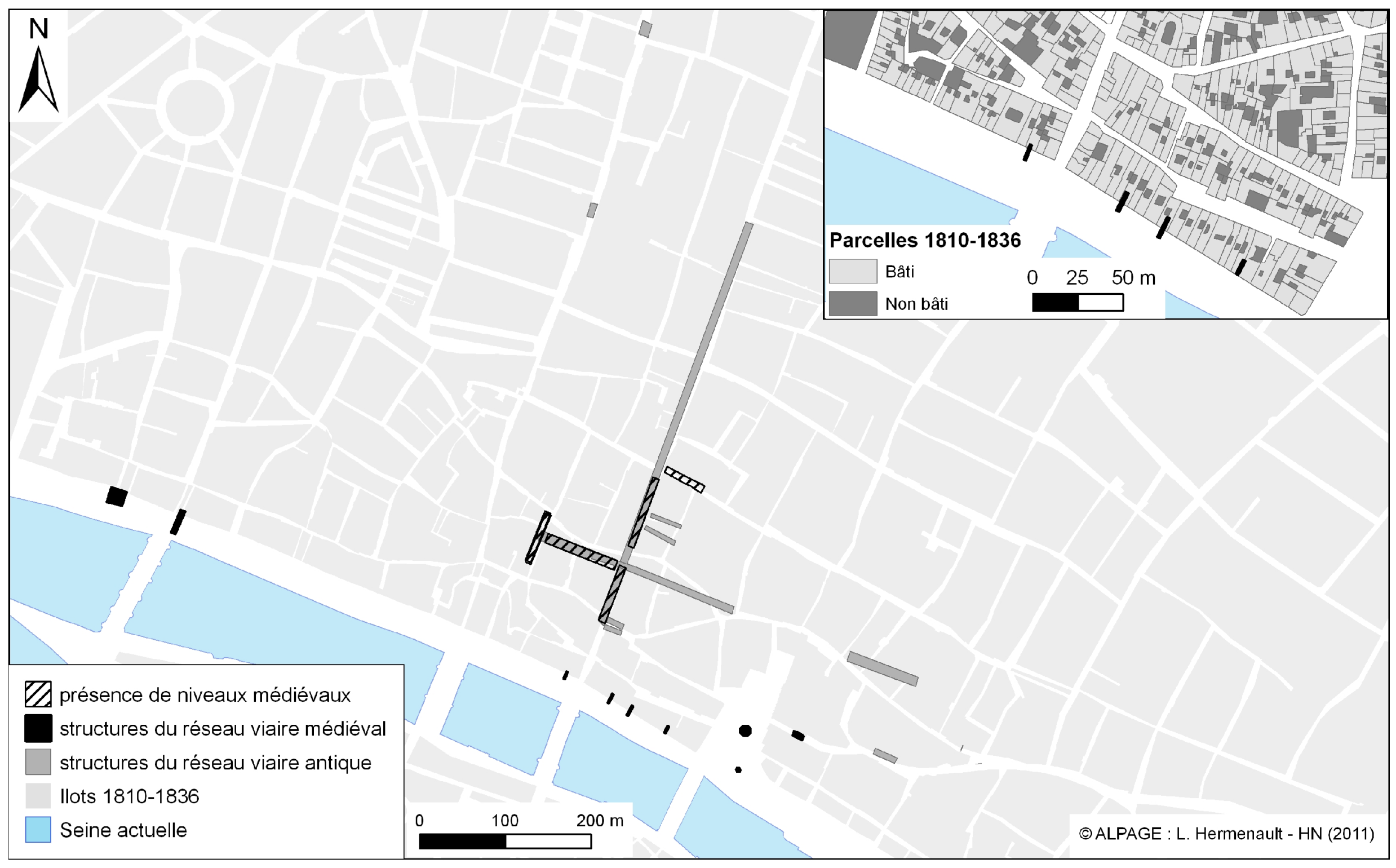

3. Structures archéologiques des réseaux viaires antique et médiéval 


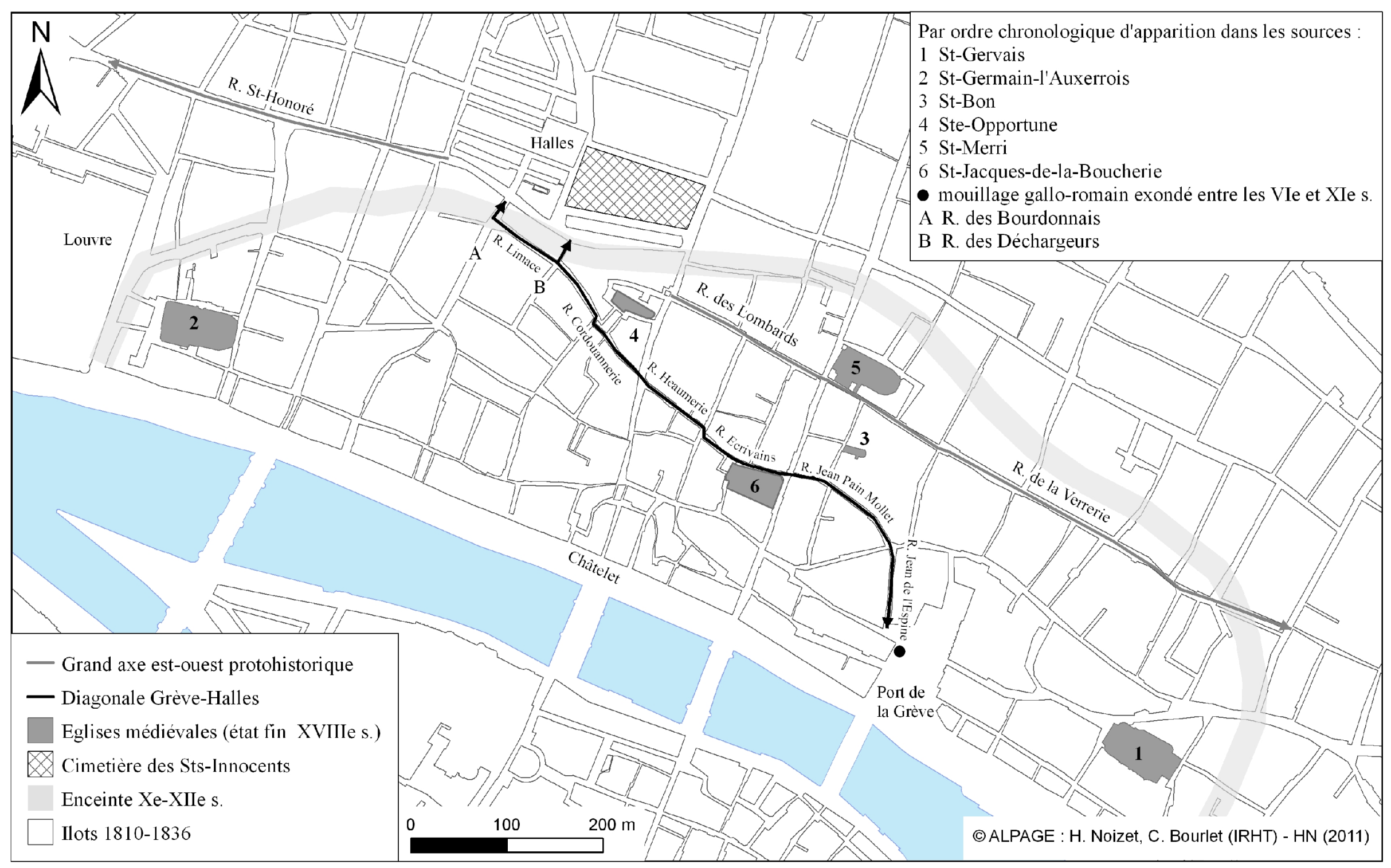

4. Principaux tracés viaires en rive droite 


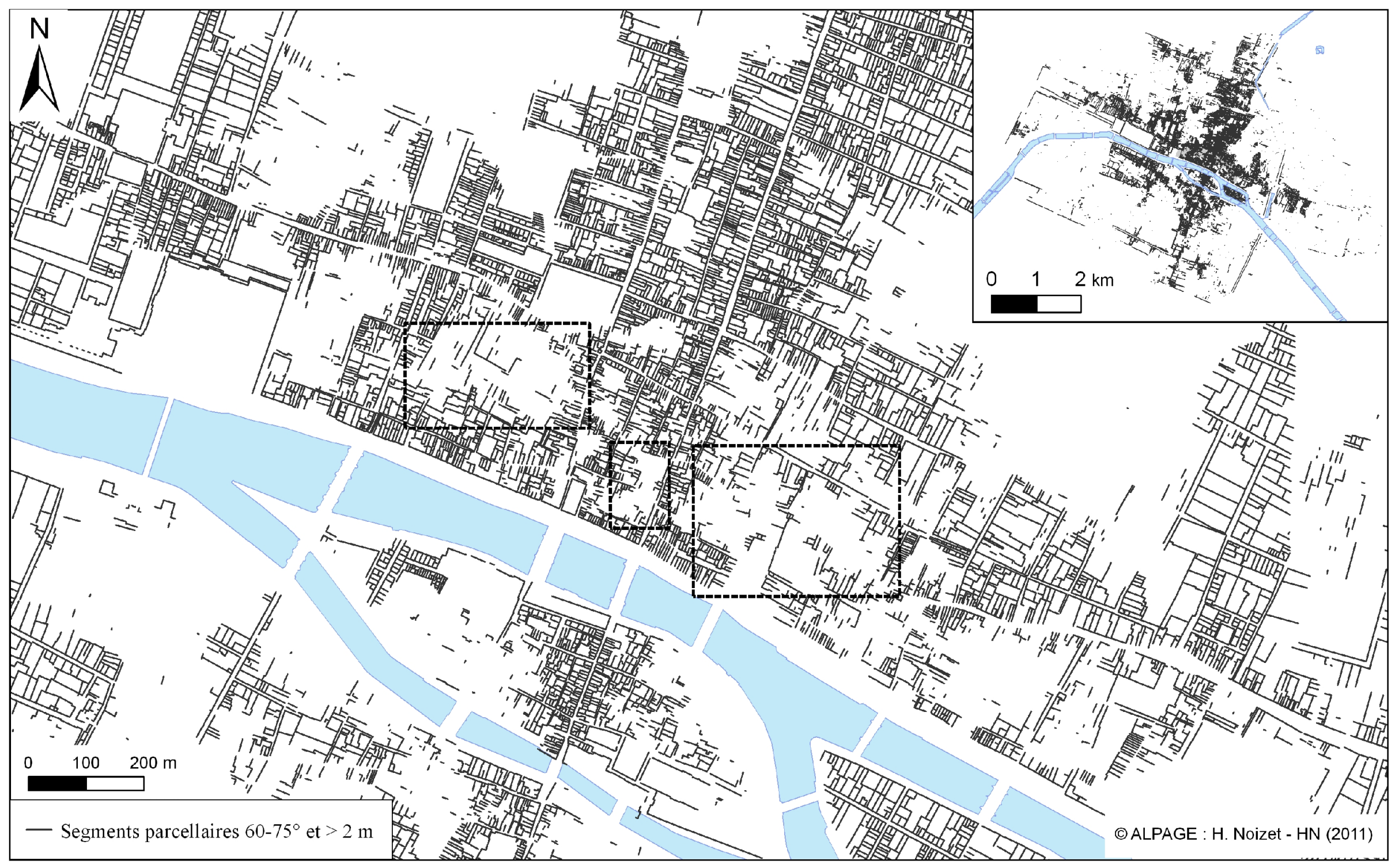

5. Rupture de la trame parcellaire héritée de l'Antiquité sur la rive droite 


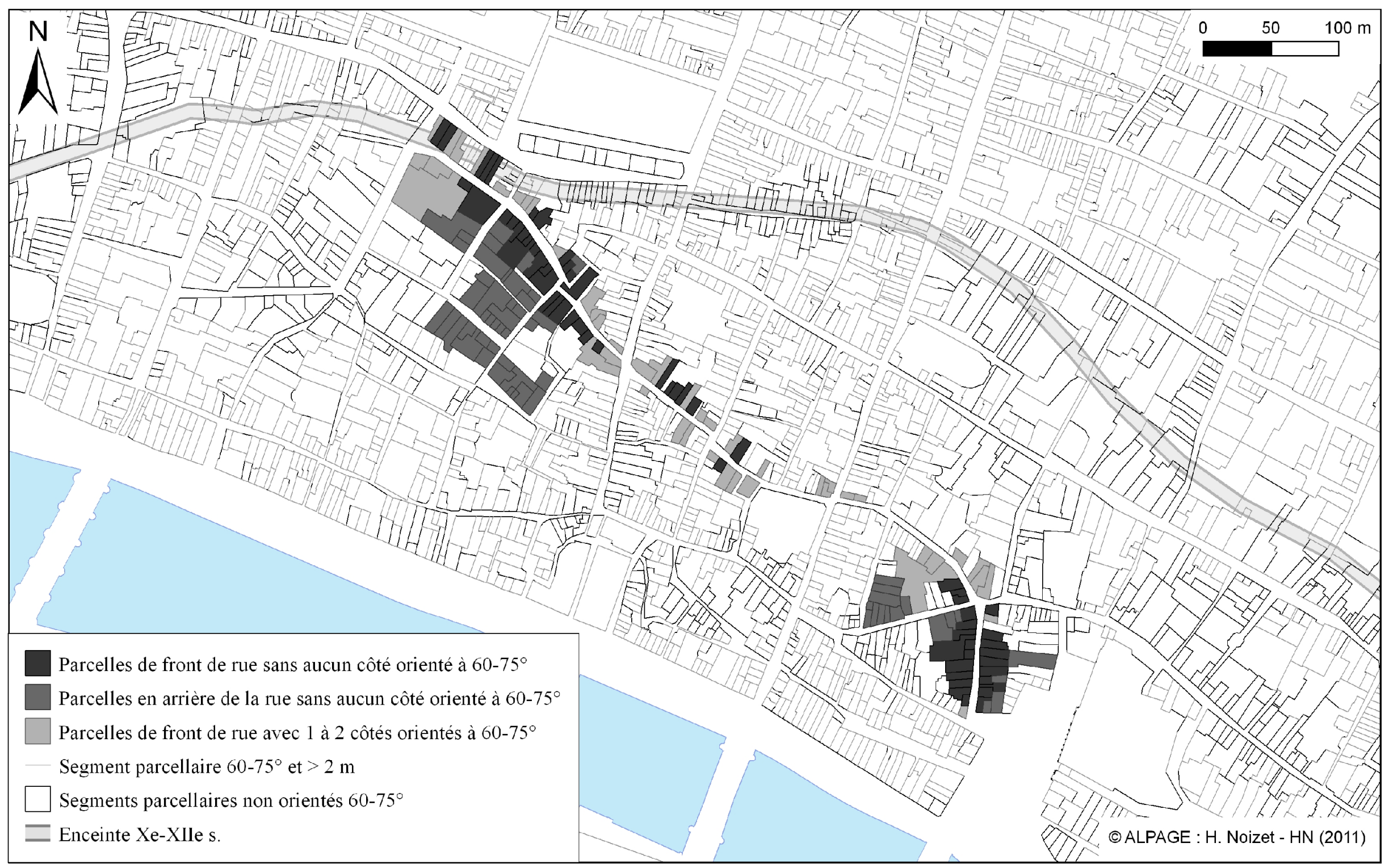

6. Discordance parcellaire de l'axe Grève-Halles 